\title{
تحليل مقرر اللغة العربية للطلاب الجدد
}

\author{
إعداد \\ حياة الفضيلة بلونجان \\ مخلصا
}

\section{ملخص البحث}

أن هذا البحث يبين عن مقرر اللغة العربية المستخدم في الجامعة الإسلامية الحكومية بادنج سديمبوان كما عرف، كان في تعليم اللغة العربيةيستخدم الكتاب الذي غير مناسبة

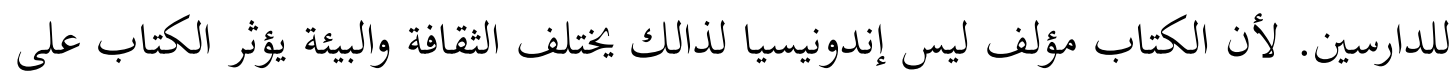
عملية التعليم المقرر هوعنصر واحد في المناهج التعليمية يعطي هذا الكتاب بحموعة واسعة من الكتب وأدوات الدعم في عملية التعلم، مثل الأشرطة أوالأقراص المدبحة والكتب الإملاء

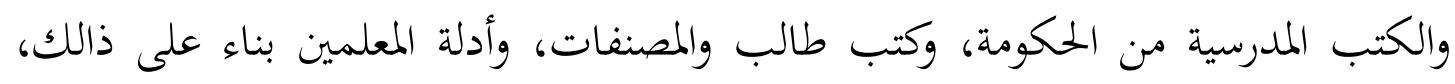
فالهدف من هذا البحث لتحلل انسجام المقرر المستخدم في الجامعة الإسلامية بادانج سيديمبوان بالأسس تصميم لمعرفة المادة المقرر المستخدم في تعليم اللغة العربية في الجحامعة الإسلامية الحكومية بادنج سيديمبوان

يجري هذا البحث في الجحامعة الإسلامية الحكومية بادانج سديمبوان وتستخدم الباحثة

تقنيتان، هما الوثائق والمقابلة يعنى تقابل الباحثة المدرس اللغة العربية خاصة وأيضا تحلل الوثائق يعنى المقرر المستخدمة في عملية التعليم وهذا البحث هوبكث النوعي وأماالطرق المستخدم في هذالبحث هوالمنهج الوصفي من البحوث التيتهد فالتقليم صورة حقيقية لما لها بحتة وفقا للسياق البحث 
وبعد ما تقوم الباحثة فحصلت النتائج البحث يتضمن المقرر المواد عما يتعلق باللغة العربية وبعضهن مفهوم سهلا يتحدث عن الترويح عن الترويح عن النفس والمهن والجوائز وغيره أن هذا الكتاب تناسب للدارسين من وجهة ثقافة، وعلم النفس فيها وحدة تتحدث عن الحياة الزوجية وغيرها ويهتم المقرر بمهارات اللغة منها مهارة الاستماع والكلام والكتابة والقراءة وعناصرها منها تراكيب.

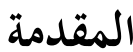

عند الخولي، أن اللغة هي نظام الصوت الذي يتكون من الرموز التعسفية التي يستخدم

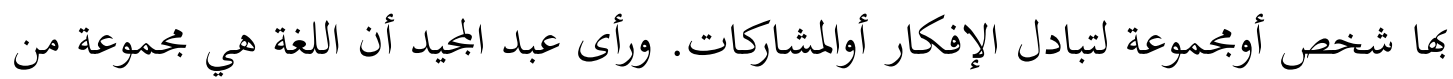
إيماءات يستخدم الناس ليعبر الأفكاروالمشاعر والانفعالات والرغبات. وتعريف الاخر، أن اللغة هي الآلة ليعبر الأفكار والحاجات والمقاصد من خلال بناء الجحملة أن تكون المفهومة لآخرين.

تعليم اللغة العربية للناطقين بغيرها هوالشئ الذي لا يمكن ان تحنبها، لأن أهمية اللغة العربية لشعوب العالم اليوم مهمة جدا سواء كان لمسلم أوغيرهم. يؤكد ذلك من خلال مؤساسات تعليم اللغة العربية في مختلف البلدان، منها الجحامعة الأمريكية في مصر ومعهدم الدراسات الإسلامية في مدريد اسبانيا والمعهد الشملا في لبنان والمركز خرتوم في السودان ومعهد العلوم الإسلامية والعربية قي إندونيسيا في جاكرتا والمعاهد تعليم اللغة العربية وتملكها مؤسسة الخيري لدولة الإمارات التي إنتشرت في إندونيسيا، منها في سورابايا وماكاسر ومالانج وباندونج وسولووالمعاهد في إنحاء البلاد.2 hlm. 9

\footnotetext{
${ }^{1}$ AcepHermawan, MetodologiPembelajaranBahasa Arab (Bandung: PT RermajaRosdakarya, 2011),
}

${ }^{2}$ Ibid., hlm. 99 
أنشطة التعليم هي عملية التفاعل بين الأفراد، سواء كان بين معلم والمتعلم، أوبين المتعلم والمتعلم. في عملية التعلم، هناك يسمى المنهج الدراسية. ويتكون على عناصر، منها: المقرر والعماليات ووسائل وأساليب التعليم. أما المقرر هوأساس في كل مستوايات التعليم،

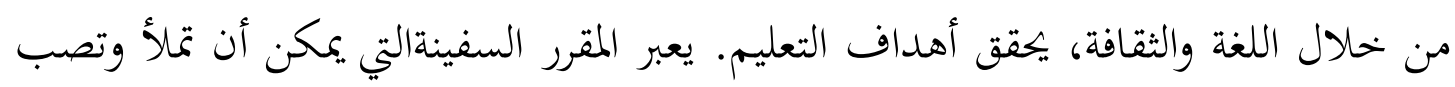
به في "إناء" الطلاب الذين عاطشون، هل هوسيمال الغذاء الطيبة ولذيذة أوالشراب المر الأليم. معلم كوسيلة أوالوسيطة الذي يملأ محتوايات الاسفينة إلى المتعلمين. الوسيلة والوسيطة يستخدم في بعض الأحيان، واحيانا لم توجد فيه. فوجب علينا أن غتم محتواياته اهتماما كبيرا فهوالمقرر. وجد فيه الأهمية الخاصة في عملية التعليم، وأيضا من عناصر الأساسية ولها دور كعمل محفز للمعمين والطلاب، ولها أيضا ألسلبية للطلاب وأيديولوجتهم، لذالك المقرر كسكين ذوحدين، واحيانا ايضا لها إيجابي كما سلبي اذا لم يستعد تماما. أولم يتم على أساس المبادئ أولم يتناسب مع المرجوة البحتمع والدين. وذلك أهمية المقرر، وخحاصة لتعليم اللغة العربية للناطقين بغيرها. في العالم التعليم في إندونيسيا خحاصة لتعليم اللغة العربية يستخدم المقرر عربية الذي يؤلف ليس إندونيسيا. لذالك فتختلف ثقافة وبيئة المقرر وإندونيسيا.

ولذلك، الباحثين المهتمين في رفع عنوان"تحليلمقرر اللغة العربية للطلاب الجدد "

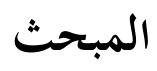

\section{أ. العربية وخصائصها}

اللغة العربية هي احد اللغة التي تتطور بتطوير الإجتماعية والعلوم. ومتى إنتشرت اللغة العربية في المجتمع المستخدمي؟ يختلف اللغوين عن هذه المسئلة. يرى بعضهم انتشرت اللغة العربية وتطورت كما اللغة الأخرى. كما عرفنا انتشرت اللغة وتطورت بالإشارة وتقليد أصوات حولما. منها: نسمة وصاعقة وخر الماء وغيرها. والأخريات، وفق أن اللغة 
هي إلهام من الله سبحانه وتعالى، يعنى يولد شخص وهوا يجلب طقة اللغة. وبعضهم، يرى

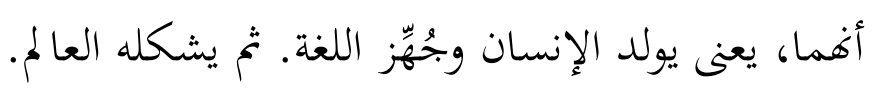
وأما خصائص اللغة العربية ولم بنحد في اللغة الأخرى منها:

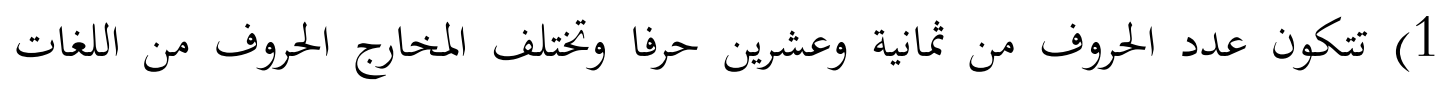
الأخرى.

2) الإعراب، هوشيء الذي يضع في هاية الكلمة وأحوال المعينة. سواء كان رفع ونصب وخفض وجزم اسما وفعلا.

3) علم العرض وبه تطور الشعر تطويرا كاملا.

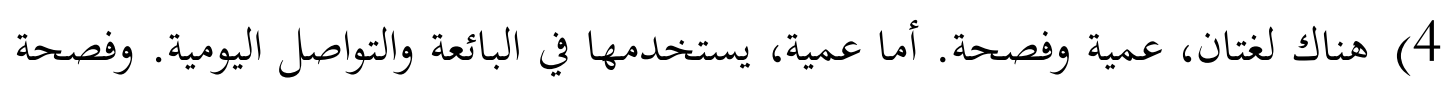
هي لغة الأدب والتعليم. وهي اللغةالرسميةالمستخدمةفيالكتابالإسلاموالعلم. 5) هناك حرف "ض" ولم لمند في اللغات الأخرى. 6) تنغير جملة الفعلية تتعلق بعمل الفعل.

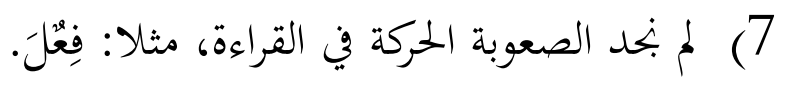
8) ملم يلتقيا حرفان في الكلمة واحدة مباشرة. 9) عدم الكلمة تتكون على حرفين ولكن أكثر منه ثلاثة أحرف ثم يزيد حرفا أوثانيا أوثلاثة واربعة أحرف.

10) عدم شكل على اربعة احروفمستمرووأيضا الجوانبالأخرالمدرجةقفينطاقبناء الداخلي وارئي منحيثابلماز وعلمالأصوات والقاموس.

1اللغة العربية هي مرنة، وتمسك القياس، وكثير من اشتقاق والمفردة.3 3

\footnotetext{
${ }^{3}$ Abd. Wahab Rosyidi dan Mamlu'atul Ni'mah, Memahami Konsep Dasar Pembelajaran Bahasa Arab (Malang: UIN-Maliki Press, 2011), hlm. 5
} 


\section{ب. مفهوم الأساسية لنظرية اللغة وتعليمها}

هناك نوعان مهمتان من مفهوم النظري اللغة، هما: التدفق الهيكلي والتحول التوليدي.

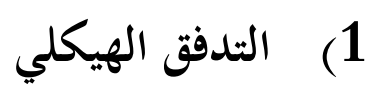

قدم السويسريفرديناندديسوسير هذا المذهب (1857-1913) ثم يطور ليونارد بيلومفيد. وهويضع أساس اللغوية الهيكلي على البحث طريقة البحث العلمي. وهناك نظريات بالنسبة لهذا المذهب، منها:

اللغة هي الكالام.

$$
\text { (ب) حصل على مهارات اللغة تعودا وتدريبا وامتحانا. }
$$

(ت) لكل اللغة لها نظام مختلف، لذالك لم تحصل على تحليل اللغة باستخدام مبني اللغة

$$
\text { الأخرى. }
$$

(ث) ولكل اللغة لها نظام كاملا وكافيا ليعبر غرض المتكلم. لذالك، لا احد من اللغات

$$
\text { أحسن وافضل من اللغات الأخرى. }
$$

(ج) تتطور اللغات بتطوير الزمان أوالوقت، لأن اللغة تتصل لغة الأخرى أواللغات. فيتغير

$$
\text { القواعدها. }
$$

(ح) مصدر الأولى والأساسى اللغة هي متكلم، ليس مؤسسة العلمية ومركز اللغة أومدارس

$$
\text { النحوية. }
$$

بناء على ذالك، فيرخس المبادئ لتعليم اللغة العربية، منها:

يحصل الطاقة با لتعود، فوجب أن يحافظ ويقلد مرارا وتكرارا، فا للمعلم دور الأساسي. 
(ب) اللغة هي مصدر الأساسي، فوجب على المعلم أن يبدأ الدرس بالإستماع والكلام والالقرأة والكتابة.

(ت) نتائج التقابل اللغوي فهومصدر الأساسي لتصميم المادة والإمتحانات.

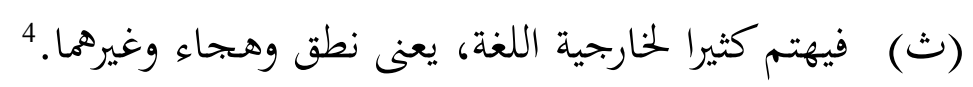

ت. تعليم اللغة العربية

تعلم اللغة العربية يختلف تماما عن لغة الأم، لذالك، الوسائل التي توصل إليها ايضا

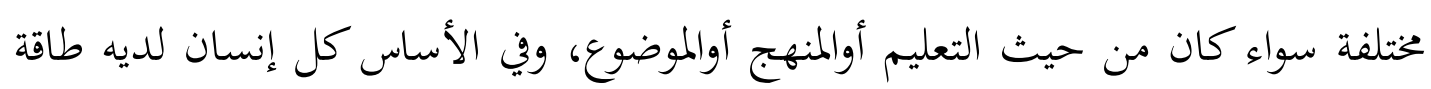

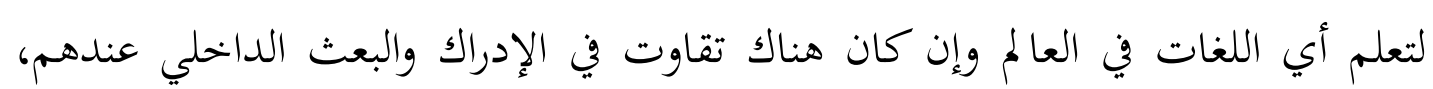

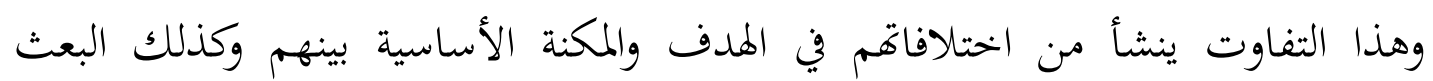
الداخلي.

كل من يتعلم العربية هؤلاء ليسوا فقط مختلفين في لغاتم الأمية بل الأهداف وما يحركهم فيه كذالك، فلكل واحد منهم له هدف معين وبه يسعى إلى تحصيله.

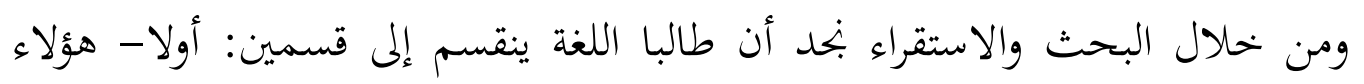

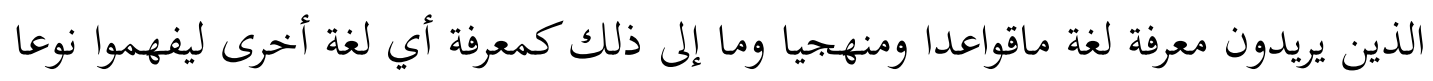
ما فيها، وهذا القسم غالبا لا يتمسك بالوثيق.

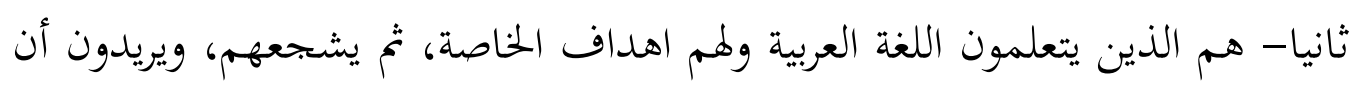
يحققوا أهدافهم ليقضى رغبتهم.

${ }^{4}$ Ibid., hlm. 7-8

${ }^{5}$ Syaiful Mustofa, Strategi Pembelajaran Bahasa Arab Inovatif (Malang: UIN Maliki Press, 2011), hlm. 115. 
عند ما نالاحظ دقة عن المناهج والمقررات في المؤسسة والمعهد تعليم اللغة العربية للناطقين بغريها فنجد خطأ في المناهج. مثلا، طلاب الدبلوماسي الذين يتعلمون اللغة المعينة ليتصل مع حولم ولكن يعطيهم مادة غير مناسبة. اويعطيهم المادة التي يعطى بكلي لبكارليوس مثلا، أوللدبلوم.

الشروط الأساسية في تصميم المقرر هي تناسب المقرر مع الطلاب، ولكن لم بحد اوعدم المقرر مناسبا للناطقين بغيرها.

في تصميم المقرر، وجب للمؤلف ان يقارن سن وذكاءوخلفية التعليم كما يقارن عن رغبتهم وأهداف لتعليم اللغة العربية.7 7

أما استرابجية التعليم هي طروق التي تستخدم المعلم ليختار عملية ستكون في عملية

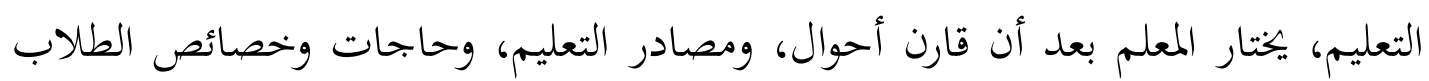
ليحقق أهداف التعليم.

مبادئ عملية التعليم، يقول بدري هي خمسة مبادئ، منها: ( ) أولوية، ؟) دقة، ؟) تدروج، §) دافع، 0) أساسي.

\section{1 ) استراتجية تعليم مهارة الإستماع}

مهارة الإستماع أولى يمر بها الطفل في اكتساب لغته الأم، وينر بها متعلم اللغة الأجنبية. ومن المعلوم أن من لا يسمع لا يتكلم، والأصم من الصغر يكون أبكما، ولذا يغلب على من فقد هاتين الحاستين أن يكون في برنامج تعليمي واحد ( لغة

${ }^{6}$ Sudi Yahya Husein dkk, Menyusun Buku Ajar Bahasa Arab ( Padang: Akademia Permata, 2012),hlm. 78

${ }^{7}$ Abd.Wahab Rosyidi dan Mamlu'atul Ni'mah, Op. Cit., hlm. 66-68 
الإشارة). الإستماع هوسبيل اولى الذي يستخدم الإنسان ليتصل بينهم في مرحلات معينة، نعرف المفردة بالإستماع والشاكلة والتراكيب.

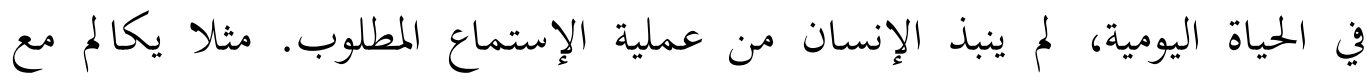
اصدقاء، ويناقش في الفصل، ويستمع التلفاز. كلهم يطلب الإستماع. مهارة الإستماع هي مهارة اللغة مهمة جدا، فوجب على الطلاب ان يمسكها. أحدى قواعد اللغة يقول أن اللغة هي كلام، يعنى أصوات مسموع ومنطوق. لذالك، الإستماع هوخبرة التعليم مهمة جدا للطلاب، لا بد اند يعطى المعلم اهتماما كثيرا. هناك ثلاثة مهارات التي سيهتم ويطور في الإستماع، منها: (أ) مهارة التعرف صوت اللغة العربية دقة.

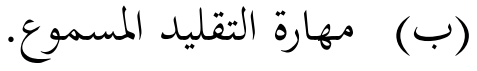
(ت) مهارة المفهوم المسموع. (ت) أما اهداف تعليم اللغة العربية، هي:

$$
\begin{aligned}
& \text { (أ) التقليد } \\
& \text { (ب) تحفيظ } \\
& \text { (ت) يستنتج الفكرة المهمة } \\
& \text { (ث) يفهم المحتوى. }
\end{aligned}
$$

وهناك ثلاثة أنواع الإسترابتية لتعليم الإستماع بإستخدام القرص المضغوط، منها: (1) بإستخدام نص مقطوع. وتمدف هذه استرابتية لتدرب الإستماع المقروء وتفهم محتوته. 
(2) استراتجية التسجيل. تركز هذه الإسترابتية على فهم مقروء وإجابة الأسئلة في كل مقروء.

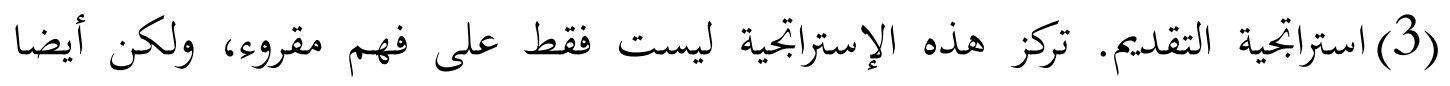

لتدريب التعبير بلغتهم.

\section{2) 2 استراتجية تعليم مهارة الكلام}

مهارة الكلام هي إحدى اللغات سيتحصل في تعلم العربية. الكلامهوالوسيلةالرئيسيةلتعزيزالتفاهمامتبادل والتواصلالمتبادل،وذذلكباستخداماللغة كوسيلة.

وأحيانا، تسمى مهارة الكلام التعبير. ومع ذالك هما تختلفان تركيزا، وتركز مهارة الكلام شفويا، وأما التعبير يركز شفويا وأيضا كتابة. لذالك في تعلم اللغة العربية بنح تعبيرا شفهيا وتريريا، وكلاهما له تشابه، يعنى مشتعل للتعبير أفكارهم. يمكن نستخدم الإستراتيحية في تعلم مهارة الكلام، منها:

استرابحية مباشرة. وتمدف هذه الإتسراتجية لتدرب الطلاب أن يعبروا ما راوا باللغة

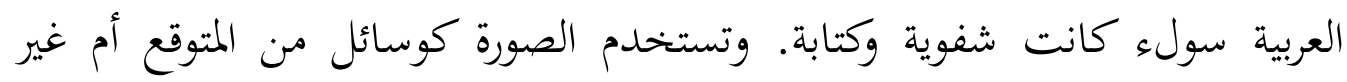
متوقع. (ب) استراتجية بانوراما. وتهدف هذه الإستراتجية على فهم المقروء كاملا عن طريقة يقسم

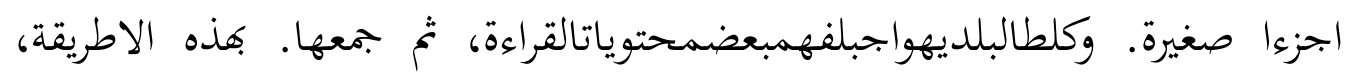
يرجوللطلاب أن يفهموا المقروء سرعة. (ت) استرابجية بجموعة صغيرة. في هذه الاستراتجية ينقسم الفصل إلى بجموعات صغيرة.

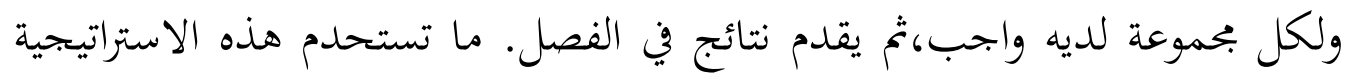
لزيادة تمكين الطلاب، حتى يتمكن كل طالب من بحربة نفس التجربة التعليمية. 
(ث) استراتيجية عرض الصور. تسمى هذه الاستراتيجية استرانتجية جلسة المعرض.يهدف هذه الاستراتيحية إلى تدريب الطلاب على فهم المقروء، ثم تصور في شكل الصورة. من الصورة المتوقعة من جميع الطلاب يمكن حفظ محتوى القراءة سهلة ويمكن الطلاب أن يذاكر المقروء لفترة طويلة.

\section{استراتيجية تعليم مهارة القراءة.}

تتكون مهارة القراءة على جانبين، هما تعيير الرموإلى الصوت واستيعاب معنى الوضع برمته برمز الكتابة. يمكن أيضا أن تتجلى القدرة على القراءة سواء كان قراءة ظهرية وصامتة، لهابل قراءة ظهرية لا يظهر فقط فهم ما يجري قراءته، وقراءة ظهرية أسهل في القياس من قراءة صامتة.

مهارة القراءة هي تقديم الموضوع بإختصاص الموضوع القراءة، ثم يتبعه الطلاب. هذه المهارة تركز على تمارين شفويا أوسرد عن طريق الفم، تدريب الفم للتحدث بطلاقة، صحيح

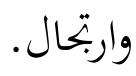
تنقسم استراتيجية تعليم مهارة القراءة إلى ثلاثة اقسام، هما استراتيجية لمرحة المبتدئ، ومرحلة المتوسط ومرحلة المتقدم. مرحلة المبتدئ. في هذه المرحلة عادة يستخدم استراتيجية طلب غخطط فارغ، يعنى استراتيجية تستخدم لتدريب مهارة الطلاب في التعبير محتويات القراءة في شكل الجدول. مثلا، يستطيع الطلاب أن يفرق بين الإسم والفعل. (ب) مرحلة المتوسطة. في هذه المرحلة عادة تستخدم استراتيجية تطابق بطاقة الفهرسة، يعنى تستخدم استراتيجية لتعليم الكلمات اوالجمل مع شريكها. مثلا، الكلمة ومعننها اوالأسئلة والإجابة، وغيره. 
(ت) مرحلة المتقدمة. في هذه المرحلة عادة تستخدم استراتيجية تحليل، يعنى تستخدم استراتيجية لتدريب الطلاب على فهم المقروء بتحليل فكرة رئيسية وفكرة دعامة.

\section{استراتيجية مهارة الكتابة}

الكتابة هي مهارة اللغة مندبحة، قهدف إلى إنتاج الكتابة. الكتابة هي قدرة ومهارة

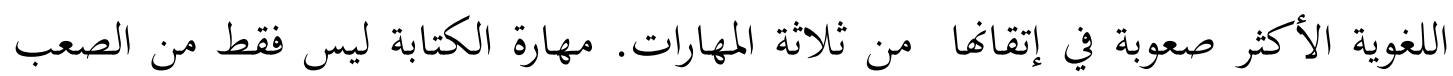
أتقاها للناطقين بغيرها، ولكن أيضا للناطقين بها. هذا يرجع إلى القدرة على كتابة أتقان عناصر اللغة مختلف وعناصر خارجها لتزين الكتابة. على أساس، الكتابة هي الأنشطة الإنتاجية والتعبيرية. في الكتابة يجب أن يكون إنهر ماهرا لاستخدام دراسة الخطط، هيكل اللغة والمفردات. تستخدم مهارة الكتابة لتدوين الملاحظات، وتسجيل ومقنع وتقرير وإبلاغ، ومؤثر القارئ. لايمكن تحقيق الغرض والهدف من هذه الادراسة إلا متعلمي اللغة تأليف وبتميع طريقة التفكير ووضعها واضحا، وطلاقة وتواصلية. هذا الوضوح يعتمد على العقل، تنظيمة واستخدام واختيار الكلمات، هيكل

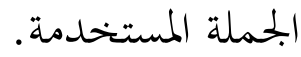

\section{ث. طريقة تعليم اللغة العربية}

كل طريقة لديه مزايا وعيوبا. وغالبا ما تولد الطريقة من عدم رضاه عن الطريقة السابقة، وفي نفس الوقت، الطريقة الجحيدة بدورها هي أيضا في الضعف الذي اعتاد أن يكون سبب ولادة الطريقة التي يتم انتقادها. طرق تأتي وتذهب مع نفس نقاط المزاي والعيوب. ومع ذالك، كل طريقة لديه دور مهمة، اعتماد على الشروط المطلوبة. في تعليم اللغة الأجنبية يوجه حالة موضوعية بين بلد وبلد أخرى، بين مؤسسة ومؤسسة أخرى، فترة 
واحدة مع آخر. وتشمل حالة موضوعية اهداف التدريس، وحالة الطالب ووسائل وبنية التحتية وغيرها.

تنقسم طريقة التعليم على ثلاثة أقسام، منها: ( ) طريقة تركيز اللغى، ؟) طريقة تركيز

$$
\begin{aligned}
& \text { التعليم، ب) طريقة تركيز الطلاب. } \\
& \text { 1 }
\end{aligned}
$$

هذه الطريقة أنتجت طرق التدريس منها: طريقة القواعد والترجمة، طريقة المباشرة، طريقة

القراءة، طريقة السمعية البصرية، طريقة المعرفية وطريقة انتقائية.

(أ) الطريقة القواعد والترجمة. هذه الطريقة مبنية على افتراض وجود منطق واحد للكون وهوأساس كل اللغات في العالم، وأن القواعد جزء من الفلسفة والمنطق. تعلم اللغة يمكن أن يعرر القدرة على التفكير المنطقي، حل المشكلة وحفظها. وهؤلأ الذين يتعلمون اللغة بهذه الطريقة على حفظ النصوص الأجنبية كلاسيكي وترجمتها لغة الأم. (ب) الطريقة المباشرة. تنشأ هذه الطريقة بسبب عدم الرضا عن نتائج تدريس اللغة بطريقة القواعد والترجمة ومرتبطة بمتطلبات الاحتياجات الحيقيقية في المجتمع. تم تطوير هذه الطريقة على اساس افتراض أن عملية تعليم اللغة الأجنبية سوى تعليم اللغة الأم، يعنى باستعمال اللغة مباشرة ومكثفة في الإتصال، بالإستماع والكلام، ثم تم القراءة والكتابة. (ت) الطريقة القراءة. عدم الرضا عن طريقة المباشرة لايهتم اهتماما تاما لمهارتين القراءة والكتابة، يشجع المعلمين واللغوين على البحث عن طرق جديدة. في ذالك الوقت ظهرة آراء بين المعلمين أن تدريس اللغات الأجنبية مع هدف إتقان جميع المهارات اللغوية مستحيل. (ث) الطريقة السمعية البصرية. تقتصر المهارات اللغوية التي تنتجها طريقة القراءة على قدرة النص لم تكتفي للاحتياجات التي تطورت في الأربعينيات. هذه الطريقة مبنية على 
عدة افتراضات، منها أن اللغة الأولى هي الكلام. لذالك يجب أن تبدأ بأصوات اللغة في شكل كلمة، وجملة ثم ينطقها قبل تعليم القراءة والكتابة. افتراض آخر من هذه الطريقة هوأن اللغة عادة. سيصبح السلوك عادة إذا تكرر مرات. لذالك يجب ونمب ان يتم تعليم اللغة عن طريق تكرار. وأيضا تختلف اللغات مع بعضهم. لذالك، اختيار المواد "يجب أن يعتمد على نتائج تحليل التقابل اللغوي، بين الأم والأجنبية. (ج) الطريقة المعرفية. تعتمد الطريقة المعرفية على تفتراض أن التعلم مفيد وأن المعرفة الواعية بالقواعد مهمة. على الرغم أت هدف التعلم هوأتقان المهارات اللغوية ولكن يتم

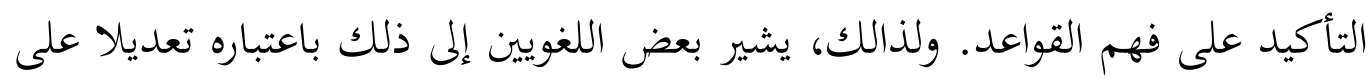
طريقة القواعد والترجمة. تظهر هذه الطريقة لأن عدم الرضا عن نتيجة الطريقة السمعية

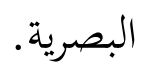

(ح) الطريقة الانقائية. الطريقة الانتقائية هي طريقة التي تستخدم أجزاء مهمة من طرق

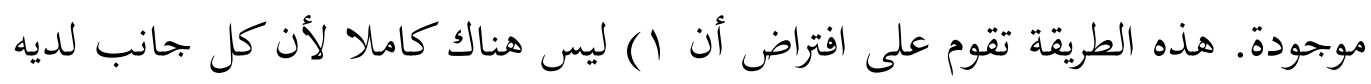

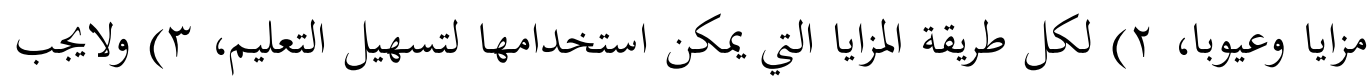

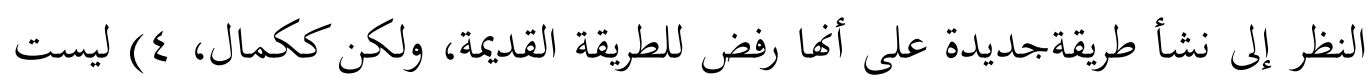
الطريقة مناسبة لكل هدف، ومعلم، وتلميذ، وجميع عملية التعليم، ه) المهم في عملية التعليم ليقضى حاجة الطلاب، ليست حاجة الطريقة، 7) لكل معلم لديه الحق والحرية في اختيار الطريقة التي تناسب المتعلم. 2) الطريقة تركيز التعليم. ظهرت هذه الطريقة حول التعليم في القرن سبعينيات، يبدوأن هذه الطريقة يهفزه تطوير بحوث تعليم اللغة الأجنبية وهي روح لإظهار التعلم المبتكر. 
الطريقة الاستجابة الجمدية. تستخدم هذه الطريقة نظرية اللغى البنيوية التي ترى اللغة كجزء من القواعد، ومع ذالك فإن تعليم اللغة الأجنبية هي نفس تعليم اللغة الأم، يعنى ان يفهم الطلاب فبل أن يتمكنوا من التعبير عن اللغة نفسها. في عملية التعليم

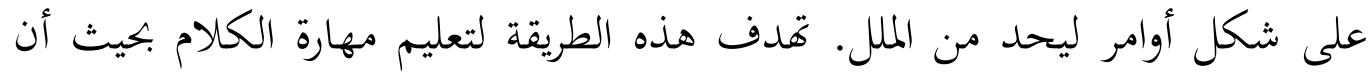
يتكن الطلاب من التواصل مع الناطقين الأصليين دون خجل أوالأحراج. (ب) الطريقة الصامتة. ظهرت هذه الطريقة على افتراض أن كل عمل مع مصادر معرفة الذات ( عاطفة، المعرفة العالمية) وليس من لآخرين، لأفم مسؤلون عما يتعلمون. وتسمى هذه الطريقة الصامتة، لأن المعلم أكثر من التحدث أثناء عملية التعليم. (ت) الطريقة التعليم المشورة. وهذه الطريقة يقدم تشارلز كوران في عام 1975، علماء النفس ويأخذ التخصص المشورة. وترى هذه الطريقة فإن ما يتم تعلمه من قبل البشر هوبشكل عام المعرفية والفعالة. (ث) الطريقة العلمية. هذه الطريقة تقوم على افتراض أن يمكن للطلاب في حالة الفصل لتعليم الكلام اللغة الأجنبية. المبدأ الرئيسي لهذه الطريقة هوا تركز بداية تعليم اللغة على لهيك اكتساب القدرات التواصلية بدلا من الكمال القواعد وأن المعلم يعطى الفرصة للطلاب في اكتساب اللغة أكثر من إجبارهم على تعلمه. (ج) الطريقة الإحائية. هذه الطريقة هي طريقة التي تنطبق على اقتراح لعلم التربية، وضعت

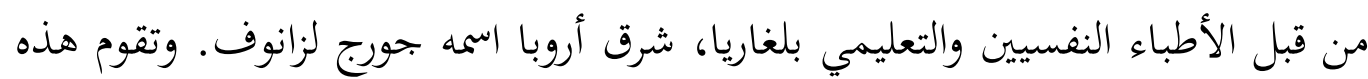
الطريقة على افتراض أن تقنيات الاترخاء والتركيز سوف تساعد الطلاب على توليد الموراد اللاوعي وتخزين بنية أكبر للمفردات. 3) الطريقة تركيز الطلاب 
تتضمن هذه الطريقة هي طريقة التواصل. تنشأ طريقة التواصلية بسبب عدم رضاهم

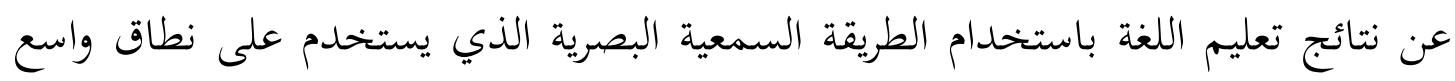

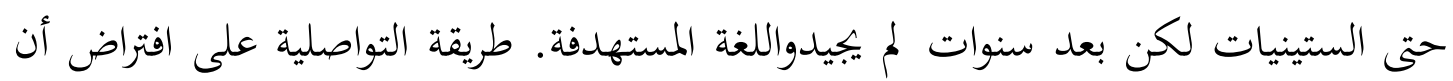
كل إنسان لديه قدرة التواصلية فطرية تسمى اداة اكتساب اللغة. 1. مقرر اللغة العربية للجامعة الإسلامية أ) - (أ) تعريف المقرر

المقررهوعنصرواحدفيالمناهجالدراسية،بالإضافةإلبعناصرأخرى،مثلالعملياتووسائلاإعلاموأ

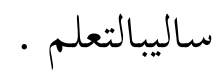

إنالكتاب المدرسي يشمل مختلف الكتب والأدوات المصاحبة، والمذكرات، والمطبوعات التي توزع على الطلاب، وكرسة التدريبات، وكرسة الاختبار الموضوعية ومرشد المعلم.9

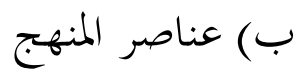
يتكون المنهج بمفهومه الحديث من أربعة عناصر أساسية هي: 1 الأهداف التعليمية

الأهداف التعليمية هي العنصر الأساسي من عناصر المنهج لأن جميع النعاصر

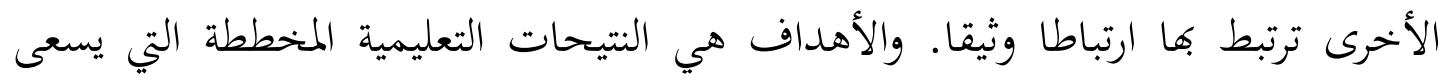

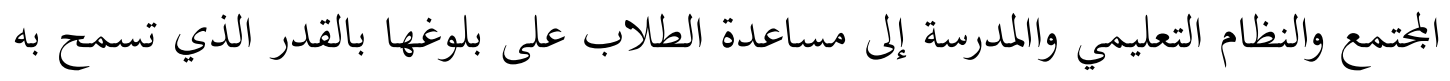

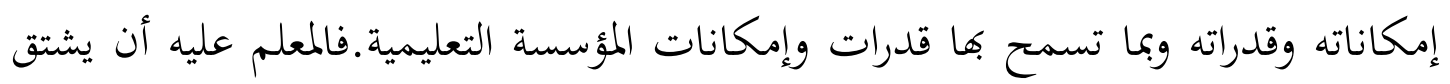

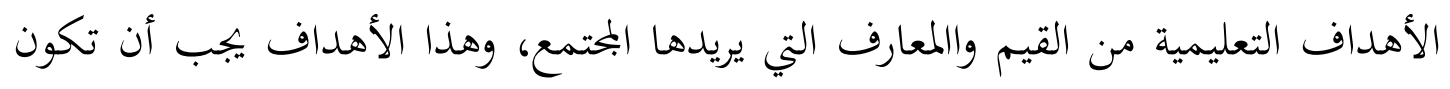

\footnotetext{
${ }^{9}$ Sudi Yahya Husein, Op. Cit., hlm. xi
} 
ملبية للاجة المختمع والطلاب الذين يتولى مسؤلية تنطيم تعليمهم في إطار عقيدة الأمة وقينها ومثلها العليا قعاداتما السليمة.

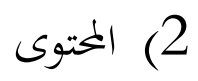
ويقصد بالمتوى المقررات الدراسية وموضوعات التعلم، وما تحتويه من مفاهيم وحقائق

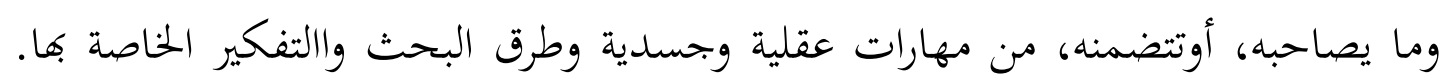

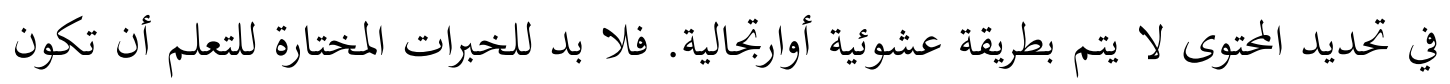
خبرات هادفة وخططة ومبنية على بحموعة من الأسس والمعايير. 10 3) طريقة التدريس هي كيفية التدريس وتختلف طريقة التدريس باختلاف الخمتوى التعليمي الذي يتم اختياره والمواقف التعليمية. 4) التقويم التقويم هوالوسيلة التي بنمع بها الأدلة عن مدى صحة الفروض التي تستند عليها

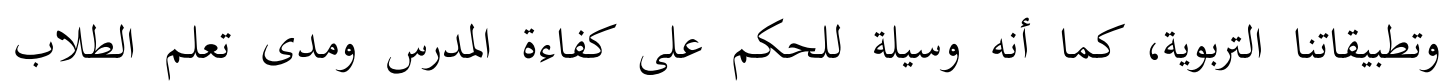

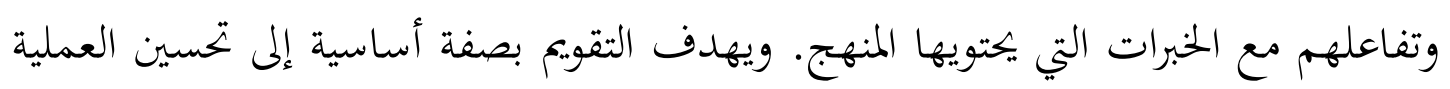
التعليمية. من فوائد فلسفة التعليمية هي:

(أ) يمكن فلسفة التعليمية تحديد الا تحاة الذي سيتم اتباعه حيث يذهب الأطفال من

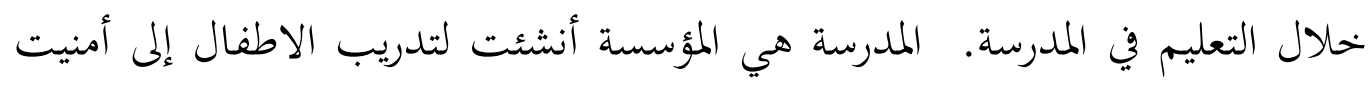
البحتمع والأمة والدولة.

10 عمد مالك سعيد، مدخل الى المناهج وطرق التدريس (الرياض: دار اللواء للنشر والتوزيع، 1415 هـ) ص 23. 
(ب) مع هدف التعليم بالفلسفة المعتمدة، نصور واضحا على حصل سيحقق. ما يجب

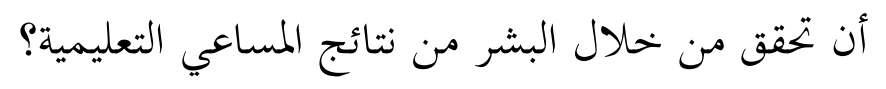

(ت) فلسفة وهدف التعليمية هما يعطيان الوحدة الموحدة لجميع جهود التعليمية.

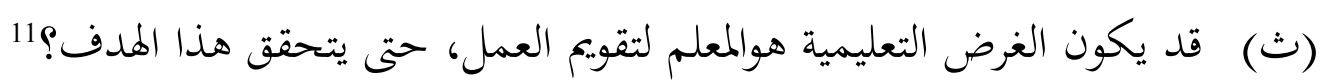
المنهج حقيقة هوآلة لتحقيق هدف التعليمية. لأن الهدف تئٔر به كثيرا فلسفة،

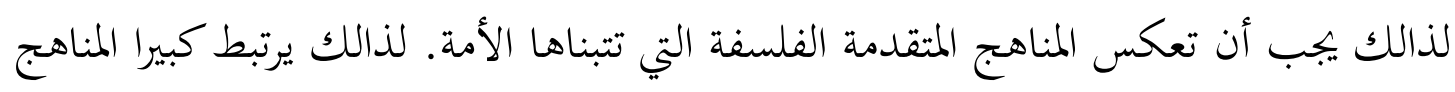
والفلسفة.

\section{الخلاصة}

1. المقرر المستخدمة في عملية التعليم اللغة العربية للطلاب الجدد في الجامعة الإسلامية

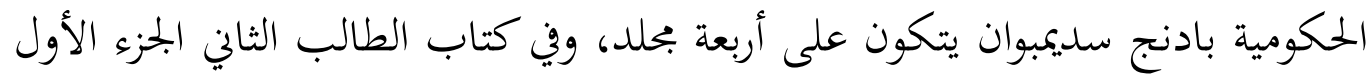
مضمون المادة العناية باصحة، الترويح عن النفس، الحيياة الزوجية، الحياة في المدينة،

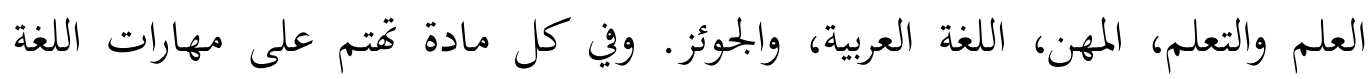

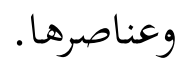

2.

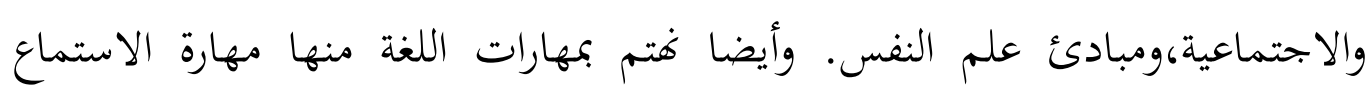
والكلام والقراءة والكتابة وعناصرها منها الأصوات والحروف والمفردات والتراكيب.

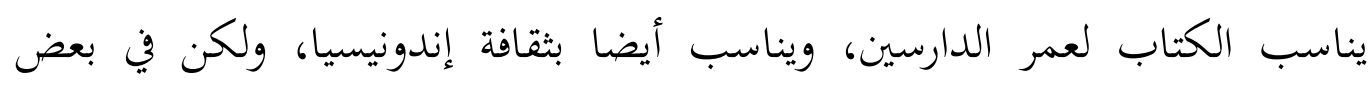

\footnotetext{
${ }^{11}$ Tim Pengembang MKDP Kurikulum dan Pembelajaran, Op. Cit., hlm. 18
} 

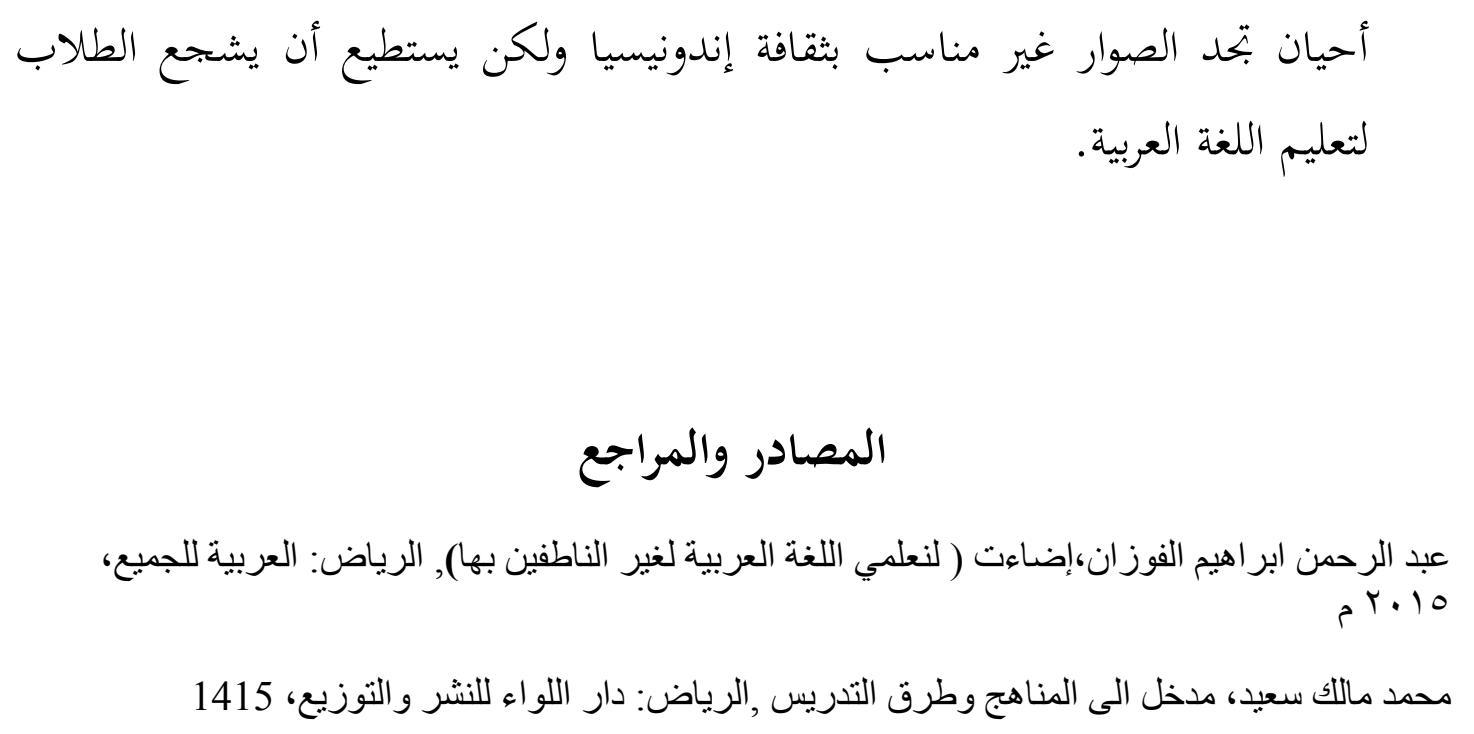

AcepHermawan, MetodologiPembelajaranBahasa Arab, Bandung: PT Rermaja Rosdakarya, 2011.

Abd. Wahab Rosyidi dan Mamlu'atul Ni'mah, Memahami Konsep Dasar

Pembelajaran Bahasa Arab, Malang: UIN-Maliki Press, 2011

Syaiful Mustofa, Strategi Pembelajaran Bahasa Arab Inovatif, Malang: UIN Maliki Press, 2011

Sudi Yahya Husein dkk, Menyusun Buku Ajar Bahasa Arab, Padang: Akademia Permata, 2012 